\title{
ANÁLISIS DE PÉRDIDAS ENERGÉTICAS Y ECONÓMICAS POR TRANSPORTE DE VAPOR EN TUBERÍAS SIN UN ADECUADO AISLAMIENTO TÉRMICO
}

\section{ANALYSES OF ENERGETIC AND ECONOMIC LOSSES FOR THE STEAM TRANSPORT BY PIPES WITHOUT AN ADEQUATE THERMAL INSULATION}

\section{RESUMEN}

Se presenta el cálculo de las pérdidas de recursos energéticos y económicos por el deterioro o ausencia de aislante térmico en las tuberías de transporte de vapor al interior de una empresa dedicada a la producción de licores, así como la predicción de ahorros alcanzados al aplicar aislantes en zonas detectadas como críticas. Se utilizan modelos de transferencia de calor para sistemas radiales y cálculos económicos a partir de costos de aislantes térmicos, recursos másicos y energéticos, y eventuales ahorros alcanzados por la implementación de aislantes. Se encontró que las pérdidas energéticas pueden reducirse hasta en un 99\%, con rápida recuperación de los costos de inversión según la selección del aislante. Los modelos presentados pueden ser adaptados por otras empresas que deseen evaluar de manera rápida y efectiva los eventuales ahorros en costos de producción derivados de la minimización de pérdidas energéticas a través de sus tuberías.

Palabras clave: aislamiento térmico, perdidas de calor en tuberías, transferencia de calor, tuberías de transporte de vapor.

\begin{abstract}
The calculation of the loss of mass energy and economic resources for the deterioration or absence of thermal insulation coating in the steam pipelines of a company devoted to the production of spirits is presented. It is also presented the resource savings achieved by the application of thermal insulation in the Company areas identified as critical. The mathematical models for heat transfer are given for radial systems. The economic calculations were made from contributions of manufacturers of insulating thermal material, as well as cost of energy resources and the estimated savings achieved by the implementation of the thermal insulator. It was found that energy losses can be reduced by up to $99 \%$, with rapid recovery of the investment costs from the proper selection of insulation. Calculation models presented in this work can be adapted by other companies that wish to assess quickly and effectively possible savings in production costs resulting from the minimization of energy losses through their pipelines.
\end{abstract}

Key words: heat transfer, pipelines heat losses, steam transport pipelines, thermal insulation

\section{Aristizábal Carlos}

Magister en Ingeniería de Química Investigador Bioprocol SAS, Grupo CERES

Departamento de Ingeniería Química

Universidad de Antioquia

caristizabal@bioprocol.com

Medellín, Colombia

\section{Schäfer German}

Magister en Gestión de Ciencia

Investigador Bioprocol SAS, Grupo CERES

Departamento de Ingeniería Química

Universidad de Antioquia

gschafer@bioprocol.com

Medellín, Colombia

\section{Barrera Rolando}

Doctor en Ingeniería. Mágister en Ciencias Químicas

Departamento de Ingeniería Química

Universidad de Antioquia

rolando@udea.edu.co

Medellín, Colombia

Tipo: Artículo de investigación

Fecha de Recepción: Octubre 7 de 2013

Fecha de Aceptación: Diciembre 22 de 2013 


\section{INTRODUCCIÓN}

En muchas industrias el vapor representa un recurso importante debido a su valor energético, económico y operacional. En diversas empresas se utiliza en múltiples procesos que incluyen destilación, fermentación y transporte de fluidos viscosos, tal y como ocurre con las melazas usadas en la fabricación de licores. Para la generación del vapor, usualmente se consumen altas cantidades de combustibles (típicamente gas natural, ACPM o biomasa) y de agua en calderas, incurriéndose además en costos asociados al ablandamiento de agua de calderas y mantenimiento en general. Debido al alto costo que puede representar para una organización o empresa el consumo de recursos energéticos, que en muchos casos pueden representar un porcentaje significativo de los costos operativos, es indispensable implementar políticas de gestión energética al interior de las organizaciones para reducir dichos consumos.

El artículo 348 del Estatuto de Seguridad Industrial (Resolución 02400 de 1979) [1] establece que las líneas que transporten o conduzcan sustancias calientes como vapores, gases o líquidos (mayores a $100{ }^{\circ} \mathrm{C}$ ) deben estar provistos de cubiertas aislantes, en especial si atraviesan o están cerca de zonas de trabajo. De lo anterior y tomando como caso de estudio una fábrica de licores para la cual se tuvo acceso a su proceso de generación y transporte de vapor, en este estudio se analizan los costos de cambiar o instalar aislantes en las tuberías de transporte de vapor que permitan garantizar la seguridad industrial al interior de la fábrica y lograr el uso racional y eficiente de los recursos energéticos [2].

El objetivo del presente trabajo, además de facilitar alternativas de reducción de costos operacionales para la Empresa interesada, pretende mostrar de forma comprensiva los modelos de cálculo de modo tal que puedan ser fácilmente adaptados por otras Empresas o compañías que deseen evaluar de manera rápida y efectiva los eventuales ahorros en costos de producción derivados de la minimización de pérdidas ener- géticas a través de sus tuberías.

\section{METODOLOGÍA: ANÁLISIS MODELACIÓN MATEMÁTICA} (1).

$$
\frac{1}{r} \frac{d}{d r}\left(k r \cdot \frac{d T}{d r}\right)=0
$$

Donde $k=$ conductividad térmica, $r=$ radio $\mathrm{y}$ $T=$ temperatura .

La rapidez a la que se conduce la energía a través del sólido se expresa con la ecuación (2).

$$
\begin{aligned}
& q_{r}=-k A \frac{d T}{d r} \\
& q_{r}=-k(2 \pi r L) \frac{d T}{d r}
\end{aligned}
$$

Donde $A=2 \pi r L$ representa el área normal a la dirección de la transferencia de calor, con $L=$ Longitud .

La distribución de temperaturas en el cilindro (o tubería) se determina a partir de la ecuación (1) aplicando las condiciones de frontera apropiadas, considerando el fluido caliente (vapor) al interior de las tuberías. Para el caso de k constante, la doble integración de la ecuación (1) conlleva a la ecuación (3); donde las condiciones de frontera $T\left(r_{1}\right)=T_{s 1}$ y $T\left(r_{2}\right)=T_{s 2}$ originan la ecuación (4), con $T_{s}=$ temperatura en la superficie de la tubería. Al sustituir la ecuación (4) en la ecuación (2) se obtiene la expresión para la transferencia de calor, ecuación (5).

$$
T(r)=C_{1} \ln (r)+C_{2}
$$




$$
\begin{gathered}
T(r)=\frac{T_{S, 1}-T_{S, 2}}{\ln \left(\frac{r_{1}}{r_{2}}\right)} \ln \left(\frac{r}{r_{2}}\right)+T_{S, 2} \\
q_{r}=\frac{2 \pi L k\left(T_{S, 1}-T_{S, 2}\right)}{\ln \left(\frac{r_{1}}{r_{2}}\right)}
\end{gathered}
$$

De donde se obtiene la resistencia térmica, definida como la razón de un potencial de transmisión a la transferencia de calor, para el caso de la conducción radial (Rt,cond) en una pared cilíndrica, ecuación (6)

$$
R_{t, \text { cond }}=\frac{\ln \left(\frac{r_{2}}{r_{1}}\right)}{2 \pi L k}
$$

En general, la resistencia térmica en un cilindro hueco (tubería) con condiciones de transferencia de calor en la superficie, se representa de acuerdo a la figura 1 [3], bien sea para un material homogéneo (figura 1a) o un material compuesto (figura 1b).

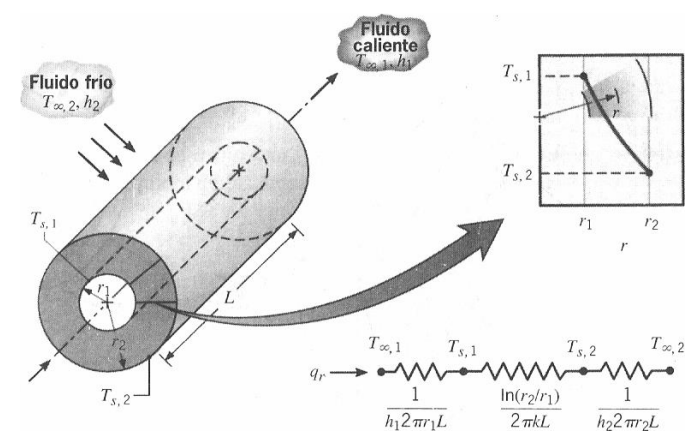

Figura 1a. Resistencia térmica en un cilindro hueco para material homogéneo [3]

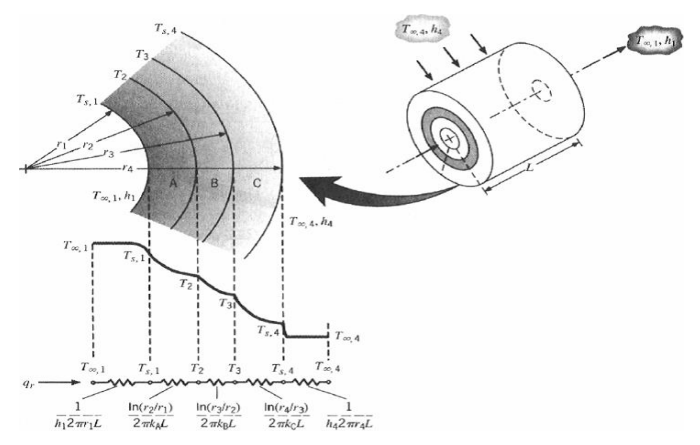

Figura 1b. Resistencia térmica en un cilindro hueco para material compuesto [3]
En la figura 1 se observa que además de la resistencia térmica por conducción $\left(R_{t, c o n d}\right)$, aparece la resistencia térmica por convección $\left(R_{t, \text { conv }}\right)$, ecuación (7) y la resistencia térmica por radiación $\left(R_{t, \text { rad }}\right)$, ecuación (8)

$$
\begin{aligned}
& R_{t, \text { conv }}=\frac{T_{S}-T_{\infty}}{q}=\frac{1}{h A} \\
& R_{t, \text { rad }}=\frac{T_{S}-T_{a l r}}{q_{\text {rad }}}=\frac{1}{h_{r} A}
\end{aligned}
$$

Con $h=$ coeficiente de transferencia de calor $\mathrm{y}$ $T_{\text {alr }}=$ temperatura de los alrededores o entorno.

El coeficiente de transferencia de calor por radiación, hr, puede calcularse con la ecuación (9)

$$
\begin{aligned}
& h_{r}=\varepsilon \sigma\left(T_{S}+T_{\text {air }}\right)\left(T_{S}^{2}+T_{\text {air }}^{2}\right) \\
& \sigma=5,6704 e^{-8}\left[\frac{W}{m^{2} K^{4}}\right]
\end{aligned}
$$

\section{Con $\sigma=$ constante de Boltzman}

Dado que las resistencias de radiación y convección superficiales actúan en paralelo, cuando $T_{\infty}=T_{a l r}$, las ecuaciones (7) y (8) pueden combinarse para obtener una sola resistencia efectiva de la superficie (ecuación (10)).

$$
q_{x}=\frac{T_{\infty, 1}-T_{\infty, 2}}{R_{t o t}}
$$

Para el caso de estudio, $q_{X}$ representa el flujo de calor a través de la tubería, $T_{\infty, 1}$ la temperatura del vapor y $T_{\infty, 2}$ la temperatura ambiente. $R_{\text {tot }}$ es el valor de la resistencia térmica total equivalente.

La resistencia térmica total para el caso en donde no se cuenta con aislante térmico en las tuberías se representa de acuerdo al diagrama de la figura 2 y se compone por una resistencia por convección $\left(R_{\text {conv }}\right)$ entre el seno del fluido y la superficie interna de la tubería, una de conducción $\left(R_{\text {cond }}\right)$ que se da a través del espesor de la tubería y por una resistencia por convección y una de radiación $\left(\mathrm{R}_{\mathrm{rad}}\right)$ entre la superficie externa de la tubería y el ambiente o los alrededores. 


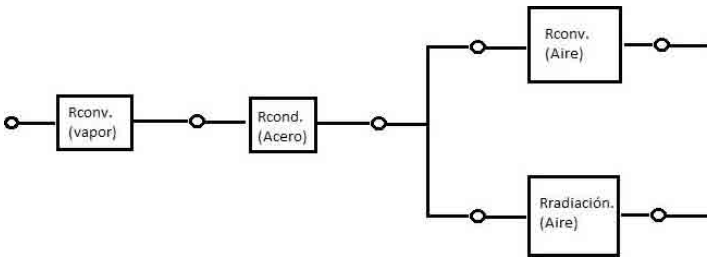

Figura 2. Circuito térmico equivalente para una tubería desprovista de aislamiento térmico

Por lo tanto el $R_{\text {tot }}$ (cuando no se cuenta con aislante térmico) puede expresarse según la ecuación (11).

$$
R_{t o t}=\frac{1}{2 \pi r_{1} L h_{2}}+\frac{\ln \left(\frac{r_{2}}{r_{1}}\right)}{2 \pi L k}+\frac{1}{h_{2}+h_{r}}
$$

Con $r_{1}$ y $r_{2}$ los radios interno y externo de la tubería.

Por otro lado, la resistencia térmica total para el caso en donde se cuenta con aislante térmico se representa por el esquema de la figura 3. En este caso la resistencia es similar a la ilustrada en la figura 2, complementándose con una nueva resistencia por conducción a través del material aislante, es decir, cuando se cuenta con aislante térmico, el $R_{\text {tot }}$ se expresa con la ecuación (12).

$$
R_{\text {tot }}=\frac{1}{2 \pi r_{1} L h_{1}}+\frac{\ln \left(\frac{r_{2}}{r_{1}}\right)}{2 \pi L k_{i}}+\frac{\ln \left(\frac{r_{3}}{r_{2}}\right)}{2 \pi L k_{\text {aisl }}}+\frac{1}{h_{3}+h_{r}}
$$

Donde $r_{3}$ es la distancia desde el centro de la tubería hasta donde termina el recubrimiento con aislante.

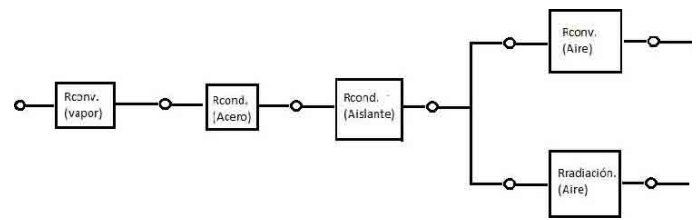

Figura 3. Circuito térmico equivalente para una tubería con aislamiento térmico

Por otro lado, los coeficientes de transferencia de calor (hi) para el interior y exterior de la tubería pueden estimarse a partir de números adimensionales como el Reynolds (Re), Prandtl $(P r)$, y Nusslet $(U n)$, de acuerdo al régimen de flujo (ecuaciones (13) - (18)) [3].

Flujo interno

$$
\operatorname{Re}=\frac{D \cdot v \cdot Q}{\mu}
$$

Flujo interno

$$
N u=\frac{h \cdot D}{k}
$$

Convección forzada flujo interno

$$
N u=0,023 \cdot \operatorname{Re}^{0,8} \cdot \operatorname{Pr}^{0,4}
$$

Flujo externo

$$
\operatorname{Re} x=\frac{u \cdot L}{v}
$$

Flujo externo

$$
N u=0,664 \cdot \operatorname{Re}^{0,5} \cdot \operatorname{Pr}^{1 / 3}
$$

Condiciones promedio, flujo externo

$$
\operatorname{Nux} \frac{h \cdot x}{k}
$$

El espesor de aislante debe seleccionarse de modo tal que permita disminuir los costos operacionales, facilitando una rápida recuperación de la inversión y al mismo tiempo proteja al personal de quemaduras por contacto con superficies calientes (superficie externa $\leq 60^{\circ} \mathrm{C}$ ) [6]. Utilizando la ecuación (19) y algún método numérico apropiado (por ejemplo bisección), se puede estimar es espesor de aislante como la diferencia $r_{3}-r_{2}$.

$\ln \left(\frac{r_{3}}{r_{2}}\right)=\frac{\left(T_{S 3}-T_{\infty}\right)}{r_{3}\left(h\left(T_{S 3}-T_{\infty}\right)+h_{r}\left(T_{S 3}-T_{a i r}\right)\right)}$

La conductividad a través la superficie externa que recubre el aislante puede despreciarse dado que su espesor es relativamente pequeño y por lo tanto no habrá diferencia significativa entre la temperatura de la superficie externa y la interna. Esta suposición puede ser compro- 
barse a partir del cálculo del número de Biot $(B i<<1)[3]$.

Por último, el periodo de retorno de la inversión (PRI) en instalación de aislantes térmicos puede estimarse con la ecuación (20).

$$
P R I=\frac{\frac{\text { Costos del aislante termico }}{\text { Ahorros }}}{\text { Periodo de operacion } \cdot \text { metro de tuberia }}
$$

\subsection{Estructura de la red de tuberías de transporte de vapor}

La red de tuberías de transporte de vapor de la empresa se utiliza para llevar el vapor generado en la zona de calderas a los diferentes procesos donde es requerido. Debido a que en la empresa se cuenta con diversidad de procesos en donde se utiliza el vapor, se tienen varios tipos de tubos que se clasifican según su diámetro nominal. Como característica general, todas las tuberías son de material acero galvanizado, por lo que su conductividad térmica, $\mathrm{k}$, $(58 \mathrm{~W} / \mathrm{mK})$, temperatura de superficie $(443,15$ K) y emisividad $(0,88)$ es similar para todas. Además se clasifican los tubos por zonas, que fueron determinadas de acuerdo a observaciones en sitio y según el avanzado deterioro o carencia de material aislante que allí se encontró en las líneas de transporte de vapor, tabla 1. La caracterización de las tuberías correspondientes se da en la tabla 2. Los cálculos posteriores de pérdida de calor y ahorros por aplicación de aislante térmico se estimaron por metro de tubería, de modo tal que con ayuda de la tabla 2 fuera posible hallar los valores o costos totales por tipo de tubo o por zona de la empresa.

Tabla 1. Distribución de zonas en la empresa

\begin{tabular}{|c|c|}
\hline Zona & Ubicación \\
\hline Zona 1 & Tanques de almacenamiento de miel \\
\hline Zona 2 & Recepción de mieles \\
\hline Zona 3 & Calderas \\
\hline Zona 4 & Terrazas cerca de procesos \\
\hline
\end{tabular}

Tabla 2. Longitud de las tipos de tuberías de transporte de vapor por zonas

\begin{tabular}{|c|c|c|c|c|}
\hline Tubo & $\begin{array}{c}\text { Zona 1 } \\
(\mathrm{m})\end{array}$ & $\begin{array}{c}\text { Zona 2 } \\
(\mathrm{m})\end{array}$ & $\begin{array}{c}\text { Zona 3 } \\
(\mathrm{m})\end{array}$ & $\begin{array}{c}\text { Zona 4 } \\
(\mathrm{m})\end{array}$ \\
\hline Tubos 1' & 0 & 14,9 & 0 & 0 \\
\hline Tubos 1,25' & 56,81 & 0 & 0 & 0 \\
\hline Tubos 2' & 0 & 69,55 & 0 & 0 \\
\hline Tubos 2,5' & 37,81 & 0 & 29,92 & 63,34 \\
\hline Tubos 6' & 0 & 0 & 30,63 & 0 \\
\hline Distribuidor & 0 & 0 & 3,67 & 0 \\
\hline
\end{tabular}

\subsection{Caracterización del material aislante}

Como base para la estimación de costos se utilizaron cotizaciones de las empresas CALORCOL [7] y SIS, Aislamientos y Refractarios [8], seleccionadas como representativas en este tipo de industria ya que, sus precios de venta de aislantes pueden considerarse valores promedio entre otras empresas del mercado. Se consideraron dos tipos de aislantes, el primero de ellos en fibra de vidrio aglomerado con resina termo resistente que le brinda una mayor eficiencia térmica y estabilidad dimensional. La presentación de este aislante para el aislamiento de líneas de vapor es en forma de "medias cañas" y acabado exterior en lámina galvanizada o acero inoxidable, incluyendo tornillos en acero inoxidable como elementos de sujeción. Según la información suministrada por el fabricante, la emisividad (superficie exterior acero galvanizado) $=0,28$; emisividad (superficie exterior acero inoxidable) $=0,85$ y conductividad térmi$\mathrm{ca}=0,0326 \mathrm{~W} / \mathrm{mK}$. Los costos de este tipo de aislante se muestran en la tabla 3 .

Tabla 3. Costo aislante $(\$ / \mathrm{m})$ fibra de vidrio sin IVA [8]

\begin{tabular}{|c|c|c|c|}
\hline Tubo & $\begin{array}{c}\text { Espesor } \\
\text { aislante } \\
\text { (pulgadas) }\end{array}$ & $\begin{array}{c}\text { Costo } \\
\text { aislamiento } \\
\text { (superficie } \\
\text { galvanizada) }\end{array}$ & $\begin{array}{c}\text { Costo } \\
\text { aislamiento } \\
\text { (superficie acero } \\
\text { inoxidable) }\end{array}$ \\
\hline Tubos 1' & 2 & 51806,4 & 61806,0 \\
\hline Tubos 1,25' & 2 & 56573,75 & 66574,0 \\
\hline Tubos 2' & 2 & 70000,0 & 90000,0 \\
\hline Tubos 2,5' & 2 & 80000,0 & 110000,0 \\
\hline Tubos 6' & 2,5 & 120000,0 & 130000,0 \\
\hline Distribuidor & 3 & 130000,0 & 140000,0 \\
\hline
\end{tabular}


El segundo aislante considerado es lana de roca, compuesto de lana mineral de roca marca stonewool, tipo manta para tubería, densidad $100 \mathrm{Kg} / \mathrm{m} 3$ y temperatura máxima de operación $750{ }^{\circ} \mathrm{C}$ y cañuela en espesor de 2 y $1 \frac{1}{2}$ pulgadas. El fabricante provee este aislante con acabado superficial de aluminio en calibre $0.7 \mathrm{~mm}$ y como elementos de sujeción incluye tornillos inoxidables $8 \times 1 / 2$ para la cubierta y alambre galvanizado calibre 18 para la cañuela de vapor y la manta. Según el fabricante, este aislante presenta emisividad (superficie exterior aluminio) $=0,07$ y conductividad térmica $=$ $0,041 \mathrm{~W} / \mathrm{mK}$. Los costos para este aislante se muestran en la tabla 4.

Tabla 4. Costo aislante $(\$ / \mathrm{m})$ en lana de roca sin IVA [8]

\begin{tabular}{|c|c|c|}
\hline Tubo & $\begin{array}{c}\text { Espesor } \\
\text { aislante } \\
\text { (pulgadas) }\end{array}$ & $\begin{array}{c}\text { Costo aislamiento } \\
\text { (superficie } \\
\text { aluminio) }\end{array}$ \\
\hline Tubos $1^{\prime}$ & 1,5 & 35304,0 \\
\hline Tubos 1,25' & 1,5 & 37783,31 \\
\hline Tubos 2' & 2 & 44511,0 \\
\hline Tubos 2,5' & 2 & 48400,25 \\
\hline Tubos 6' & 2 & 62406,00 \\
\hline Distribuidor & 2 & 57238,00 \\
\hline
\end{tabular}

Para el cálculo económico, se tomaron los precios de mercado establecidos por los prestadores de servicios públicos de los recursos básicos y energéticos utilizados para la producción de vapor, Agua y Gas Natural. Para El Agua se tiene en cuenta un costo por metro cúbico para uso industrial de $\$ 3471,1$ (COP) por el servicio de acueducto y alcantarillado (los cuales se cobran simultáneamente en la factura una vez se presenta el consumo) [9], y para el Gas Natural se toma un precio promedio por metro cúbico de $\$ 739,83$ (COP) ya que, con tal valor una de las empresas comercializadoras de los aislantes térmicos dentro de su cotización determinó los posibles ahorros energéticos y de combustibles que se podrían obtener si se hacía uso de sus productos.

\section{RESULTADOS Y DISCUSIÓN}

Los cálculos para las tuberías de transporte de vapor sin aislante térmico se realizaron con base en el análisis de una generación de 25000 $\mathrm{Lb} / \mathrm{h}$ de vapor saturado a $145 \mathrm{psi}\left(179,93{ }^{\circ} \mathrm{C}\right)$ y un periodo de operación de 222 días, tiempo promedio de trabajo de la caldera en un año. Como fluido externo se considera aire y la temperatura ambiente promedio en el sitio $25^{\circ} \mathrm{C}$.

Se evaluó la pérdida de calor, el desperdicio de gas natural (combustible de las calderas), el desperdicio de agua y las pérdidas económicas para las tuberías sin aislamiento térmico (tabla 5), las tuberías con aislamiento térmico de fibra de vidrio y superficie externa de acero inoxidable (tabla 6), las tuberías con aislamiento térmico de fibra de vidrio y superficie externa de acero galvanizado (tabla 7) y para las tuberías con aislamiento térmico de lana de roca y superficie externa de aluminio (tabla 8).

Tabla 5. Resultados de perdidas para las tuberías de transporte de vapor que no tienen aislamiento térmico

\begin{tabular}{|c|c|c|c|c|}
\hline Zona & $\begin{array}{c}\text { Perdida } \\
\text { de calor, } \\
\text { MJ }\end{array}$ & $\begin{array}{c}\text { Gas } \\
\text { Natural } \\
\mathrm{m}^{3}\end{array}$ & $\begin{array}{c}\text { Agua } \\
\mathrm{m}^{3}\end{array}$ & $\begin{array}{c}\text { Pérdidas } \\
\text { económicas, } \\
\text { (\$ colombianos) }\end{array}$ \\
\hline Zona 1 & 1001112 & 25604 & 795,3 & $21^{\prime} 702.899$ \\
\hline Zona 2 & 681356 & 17426 & 541,3 & $14^{\prime} 770.978$ \\
\hline Zona 3 & 1962888 & 50202 & 1559,4 & $42^{\prime} 553.041$ \\
\hline Zona 4 & 1093528 & 27967 & 868,7 & $23^{\prime} 706.365$ \\
\hline Total & 4738884 & 121199 & 3764,7 & $102^{\prime} 733.284$ \\
\hline
\end{tabular}

Tabla 6. Resultados de perdidaspara las tuberías de transporte de vapor con aislamiento térmico de fibra de vidrio y superficie externa de acero inoxidable

\begin{tabular}{|c|c|c|c|c|}
\hline Zona & $\begin{array}{c}\text { Perdida } \\
\text { de calor, } \\
\text { MJ }\end{array}$ & $\begin{array}{c}\text { Gas } \\
\text { Natural } \\
\mathrm{m}^{3}\end{array}$ & $\begin{array}{c}\text { Agua } \\
\mathrm{m}^{3}\end{array}$ & $\begin{array}{c}\text { Pérdidas } \\
\text { económicas, } \\
\text { (\$ colombianos })\end{array}$ \\
\hline Zona 1 & 12121 & 310 & 9,6 & 262.773 \\
\hline Zona 2 & 1697 & 43 & 11,6 & 72.509 \\
\hline Zona 3 & 4232 & 108 & 5,5 & 99.071 \\
\hline Zona 4 & 4735 & 121 & 3,8 & 102.656 \\
\hline Total & 22785 & 583 & 30,5 & 537.009 \\
\hline
\end{tabular}


Tabla 7. Resultados de perdidas para las tuberías de transporte de vapor con aislamiento térmico de fibra de vidrio y superficie externa de acero galvanizado

\begin{tabular}{|c|c|c|c|c|}
\hline Zona & $\begin{array}{c}\text { Perdida } \\
\text { de calor, } \\
\text { MJ }\end{array}$ & $\begin{array}{c}\text { Gas } \\
\text { Natural } \\
\mathrm{m}^{3}\end{array}$ & $\begin{array}{c}\text { Agua } \\
\mathrm{m}^{3}\end{array}$ & $\begin{array}{c}\text { Pérdidas } \\
\text { económicas, } \\
\text { (\$ colombianos) }\end{array}$ \\
\hline Zona 1 & 30454 & 779 & 24,2 & 660.212 \\
\hline Zona 2 & 4719 & 121 & 31,1 & 197.405 \\
\hline Zona 3 & 14994 & 383 & 13,3 & 329.993 \\
\hline Zona 4 & 18111 & 463 & 14,4 & 392.633 \\
\hline Total & 68278 & 1746 & 83,0 & $1^{\prime} 580.243$ \\
\hline
\end{tabular}

Tabla 8. Resultados de perdidas para las tuberías de transporte de vapor con aislamiento térmico de lana de roca y superficie externa de aluminio

\begin{tabular}{|c|c|c|c|c|}
\hline Zona & $\begin{array}{c}\text { Perdida } \\
\text { de calor, } \\
\text { MJ }\end{array}$ & $\begin{array}{c}\text { Gas } \\
\text { Natural } \\
\mathrm{m}^{3}\end{array}$ & $\begin{array}{c}\text { Agua } \\
\mathrm{m}^{3}\end{array}$ & $\begin{array}{c}\text { Pérdidas } \\
\text { económicas, } \\
\text { (\$ colombianos) }\end{array}$ \\
\hline Zona 1 & 186805 & 4778 & 148 & $4^{\prime} 049.704$ \\
\hline Zona 2 & 26796 & 685 & 142 & 999.479 \\
\hline Zona 3 & 73784 & 1887 & 87 & $1^{\prime} 697.452$ \\
\hline Zona 4 & 79920 & 2044 & 63 & $1^{\prime} 732.564$ \\
\hline Total & 367304 & 9394 & 440 & $8^{\prime} 479.199$ \\
\hline
\end{tabular}

Según los resultados de las tablas 5 - 8, se observa que en el caso de utilizar aislamiento térmico de lana de roca y superficie externa de aluminio (tabla 8), las pérdidas totales se reducen aproximadamente en un $92 \%$ cuando se comparan con el caso en que no se utiliza aislante alguno (tabla 5); mientras que para el caso de aislamiento térmico con fibra de vidrio usando superficie externa de acero galvanizado (tabla 6) o superficie externa de acero inoxidable (tabla 7), las pérdidas económicas se reducen alrededor del 98,4\%y 99,4\%, respectivamente.

Es bien sabido que el aislante de fibra de vidrio es más costoso que el aislante de fibra de roca, pero como muestran las tablas 5-8, con los aislantes de fibra de vidrio se logran mayores ahorros energéticos, es decir, con este tipo de aislantes se disminuye al máximo las perdidas energéticas aunque la inversión inicial requerida resulte superior. Por otro lado, si se consideran otros aspectos como la resistencia a la corrosión de los materiales que recubren el material aislante, el aluminio sería una buena opción ya que por tratarse de un material que se encuentra pasivado, se evita el desgaste del material por procesos de corrosión; no obstante, algunas casas comerciales no ofrecen recubrimientos de aluminio para aislantes de fibra de vidrio, dificultando la fácil adquisición de esta combinación de materiales. El acero inoxidable por su parte resiste de manera satisfactoria los ataques de la corrosión a diferencia de los galvanizados, los cuales se oxidan formando una superficie de óxido de hierro que deteriora y afecta negativamente las propiedades del material, como por ejemplo disminuyendo su espesor o aumentando la posibilidad de agrietamiento, mientras que los aceros inoxidables forman una película de cromo en su superficie que protege al material de la corrosión sin que su propiedades físicas y mecánicas se vean afectadas. Entre mayor sea la resistencia del material a la corrosión, se garantiza que estará sujeto a menor atención de mantenimiento o de reposición.

Otro aspecto importante de la presencia del aislante, además de disminuir las pérdidas de calor, es que mantiene una temperatura de superficie tal que no representa peligro alguno para las personas que laboran muy cerca de estas, siendo la temperatura de superficie recomienda menor o igual a $60^{\circ} \mathrm{C}$. Garantizando así, el cumplimento de la regulación existente sobre salud ocupacional y seguridad industrial en instalaciones donde se cuenta con transporte por tuberías de fluidos calientes [1,6]. En la tabla 9 se muestran los resultados de la temperatura estimada de superficie para las tuberías aplicando los diferentes aislantes analizados en este estudio. Se observa que las temperaturas de superficie son aceptables en todos los casos y para todos los diámetros de tuberías. 
Dado que con las tres posibilidades de aislamiento térmico analizadas se logran las especificaciones y proveen ahorros significativos, la escogencia del aislante a utilizar deberá sustentarse en los costos de instalación, tiempo de recuperación de la inversión, pérdida después de la instalación del aislante (económica y energética) y resistencia del material superficial que recubre al aislante térmico. Los costos de inversión y el tiempo respectivo de recupe- ración se muestran en la tabla 10 , de donde observa que la recuperación de la inversión puede ser entre uno y dos meses dependiendo del aislante seleccionado. La rápida recuperación de la inversión se debe al ahorro inmediato en combustibles y agua, además de ayudar a disminuir el eventual desgaste de las tuberías por corrosión (aunque ese aspecto no se consideró en el presente estudio).

Tabla 9. Temperatura superficial estimada para el aislante de fibra de vidrio y superficie externa de acero inoxidable

\begin{tabular}{|c|c|c|c|}
\hline Tubo & $\begin{array}{c}\text { Fibra de } \\
\text { vidrio/ } \\
\text { acero } \\
\text { inoxidable }\end{array}$ & $\begin{array}{c}\text { Fibra de } \\
\text { vidrio/ } \\
\text { acero } \\
\text { galvanizado }\end{array}$ & $\begin{array}{c}\text { Lana de } \\
\text { roca/ } \\
\text { aluminio }\end{array}$ \\
\hline Tubos 1' & 36,3 & 36,2 & 49,2 \\
\hline Tubos 1,25' & 35,1 & 35,7 & 54,4 \\
\hline Tubos 2' & 36,8 & 40,8 & 49,4 \\
\hline Tubos 2,5' & 37,0 & 41,6 & 49,2 \\
\hline Tubos 6' & 35,8 & 38,8 & 49,5 \\
\hline Distribuidor & 35,8 & 38,6 & 54,4 \\
\hline
\end{tabular}

Tabla 10. Ahorro alcanzado, inversión total y tiempo de recuperación de la inversión para las tres alternativas de aislantes analizados

\begin{tabular}{|c|c|c|c|}
\hline Aislante (recubrimiento) & $\begin{array}{c}\text { Ahorro por el uso del } \\
\text { aislamiento térmico } \\
\text { (pesos colombianos/periodo de } \\
\text { operación) }\end{array}$ & $\begin{array}{c}\text { Inversión total necesaria } \\
\text { (pesos colombianos) }\end{array}$ & $\begin{array}{c}\text { Recuperación de la } \\
\text { inversión (días) }\end{array}$ \\
\hline $\begin{array}{c}\text { Fibra de vidrio (acero } \\
\text { inoxidable) }\end{array}$ & $102^{\prime} 196.274,74$ & $23^{\prime} 599.818,98$ & 51,2 \\
\hline $\begin{array}{c}\text { Fibra de vidrio (acero } \\
\text { galvanizado) }\end{array}$ & $101^{\prime} 153.040,48$ & $20^{\prime} 820.226,98$ & 45,7 \\
\hline Lana de roca (aluminio) & $94^{\prime} 254.084,90$ & $12^{\prime} 711.233,35$ & 30,0 \\
\hline
\end{tabular}

\section{CONCLUSIONES}

La aplicación de aislante en las tuberías de transporte de vapor en la Empresa de licores conlleva a que los procesos energéticos (relacionados con el transporte y distribución de vapor) sean más eficientes, con costos de inversión que pueden recuperarse en meses ya que la implementación del aislante permite un ahorro inmediato en el consumo de gas natural y agua. Las tres opciones de aislante consideradas en este estudio garantizan temperaturas de superficie de tuberías $\leq 60^{\circ} \mathrm{C}$ que implican seguridad para el personal de planta; adicionalmente, los tres aislantes garantizan ahorros energéticos mayores o iguales al 90\% (comparados con el escenario de tuberías sin recubrimiento alguno), por lo que cualquiera de las tres opciones representa una alternativa económicamente viable para la Empresa. Considerando los costos totales, tiempo de recuperación de la inversión, temperatura externa de tuberías y resistencia de los materiales, la recomendación final a la Empresa es implementar el aislante de fibra de vidrio con recubrimiento externo de acero inoxidable. 


\section{Referencias Bibliográficas}

[1] Resolución 02400 de 1979. Ministerio de trabajo y Seguridad Social. [En línea], consultado en Noviembre 6 de 2013, disponible en: http://ley100. com/portal/riesgos/38-resoluciones/81resolucion2100 y en Febrero 5 de 2014, disponible en: http://www.ilo.org/ dyn/travail/docs/1509/industrial\%20 safety\%20statute.pdf.

[2] A. Keçebas; Determination of insulation thickness by means of exergy analysis in pipe insulation, energy conversion and management, Vol. 58, pp 76-83, 2012.

[3] P. Frank; Incropera fundamentos de transferencia de calor, 4ta edición, Editorial Prentice Hall, México, 1999.

[4] İ.T. Öztürk, H. Karabay, E. Bilgen; Thermo-economic optimization of hot water piping systems: A comparison study, Energy, Vol. 31, pp 2094-2107, 2006.

[5] A. Keçebas, M. A. Alkan, M. Bayhan; Thermo-economic analysis of pipe insulation for district heating piping systems, Applied Thermal Engineering, Vol. 31, pp 3929-3937, 2011.
[6] División Aislamientos. Fibber Glass Colombia S.A, Notas técnicas \#20 Industrial: cuanto aislamiento aplicar, Julio de 1997. [En línea], consultado en Febrero 5 del 2014, disponible en: http:// www.fiberglasscolombia.com/imagenes/ notas2/NTAisl-Ind20.pdf.

[7] Cotización 29 - 12564V2: Aislamiento Térmico líneas de vapor y condensado. CALORCOL S.A. 10 de Octubre 2011. [En línea], consultado en Octubre 11 del 2011, disponible en: http://www.calorcol. com.

[8] Cotización COT 111011: Suministro e instalación de aislamiento térmico en tubería de vapor. SIS, Aislamientos y Refractarios. Octubre 11 de 2011, [En línea], consultado en Octubre 11 del 2011, disponible en: http://www.suinso.com.

[9] Empresas Públicas de Medellín, EPM. Subdirección de Gestión Regulatoria de Agua. Tarifas para servicio de acueducto y aguas residuales. Facturación de Septiembre de 2011. 